\title{
BMJ Open HOspitalised Pneumonia Extended (HOPE) Study to reduce the long-term effects of childhood pneumonia: protocol for a multicentre, double-blind, parallel, superiority randomised controlled trial
}

Anne B Chang, ${ }^{1,2}$ Siew Moy Fong, ${ }^{3}$ Tsin Wen Yeo, ${ }^{4}$ Robert S Ware, ${ }^{5}$ Gabrielle B McCallum, ${ }^{6}$ Anna M Nathan, ${ }^{7}$ Mong H Ooi, ${ }^{8}$ Jessie de Bruyne, ${ }^{9}$ Catherine A Byrnes, ${ }^{10}$ Bilawara Lee, ${ }^{11}$ Nachal Nachiappan, ${ }^{12}$ Noorazlina Saari, ${ }^{12}$ Paul Torzillo, ${ }^{13}$ Heidi Smith-Vaughan, ${ }^{14}$ Peter S Morris, ${ }^{15}$ John W Upham, ${ }^{16}$ Keith Grimwood ${ }^{17}$

To cite: Chang AB, Fong SM, Yeo TW, et al. HOspitalised Pneumonia Extended (HOPE) Study to reduce the longterm effects of childhood pneumonia: protocol for a multicentre, double-blind, parallel, superiority randomised controlled trial. BMJ Open 2019;9:e026411. doi:10.1136/ bmjopen-2018-026411

- Prepublication history for this paper is available online. To view these files, please visit the journal online (http://dx.doi. org/10.1136/bmjopen-2018026411).

Received 3 September 2018 Revised 6 December 2018 Accepted 8 January 2019

Check for updates

(C) Author(s) (or their employer(s)) 2019. Re-use permitted under CC BY-NC. No commercial re-use. See rights and permissions. Published by BMJ.

For numbered affiliations see end of article.

Correspondence to

Professor Anne B Chang; annechang@ausdoctors.net

\section{ABSTRACT}

Introduction Early childhood pneumonia is a common problem globally with long-term complications that include bronchiectasis and chronic obstructive pulmonary disease. It is biologically plausible that these long-term effects may be minimised in young children at increased risk of such sequelae if any residual lower airway infection and inflammation in their developing lungs can be treated successfully by longer antibiotic courses. In contrast, shortened antibiotic treatments are being promoted because of concerns over inducing antimicrobial resistance. Nevertheless, the optimal treatment duration remains unknown. Outcomes from randomised controlled trials (RCTs) on paediatric pneumonia have focused on short-term (usually <2 weeks) results. Indeed, no longterm RCT-generated outcome data are available currently. We hypothesise that a longer antibiotic course, compared with the standard treatment course, reduces the risk of chronic respiratory symptoms/signs or bronchiectasis 24 months after the original pneumonia episode.

Methods and analysis This multicentre, parallel, doubleblind, placebo-controlled randomised trial involving seven hospitals in six cities from three different countries commenced in May 2016. Three-hundred-and-fourteen eligible Australian Indigenous, New Zealand Māori/Pacific and Malaysian children (aged 0.25 to 5 years) hospitalised for community-acquired, chest X-ray (CXR)-proven pneumonia are being recruited. Following intravenous antibiotics and 3 days of amoxicillin-clavulanate, they are randomised (stratified by site and age group, allocationconcealed) to receive either: (i) amoxicillin-clavulanate (80 mg/kg/day (maximum $980 \mathrm{mg}$ of amoxicillin) in twodivided doses or (ii) placebo (equal volume and dosing frequency) for 8 days. Clinical data, nasopharyngeal swab, bloods and CXR are collected. The primary outcome is the proportion of children without chronic respiratory symptom/signs of bronchiectasis at 24 months. The main secondary outcomes are 'clinical cure' at 4 weeks, time-
Strengths and limitations of this study

This international, multicentre, parallel, double-blind, placebo-controlled randomised trial will provide important new information on the long-term outcomes of children hospitalised with pneumonia who are closely followed up within a clinical trial.

- The study will determine whether a longer course of antibiotics (compared with the standard shorter 5 to 6 day treatment course) for children hospitalised with pneumonia from disadvantaged communities improves long-term clinical outcomes (reduced symptoms and signs of chronic lung disease), which addresses a large knowledge gap.

- While our inclusion criteria targets those most likely to have bacterial pneumonia, we cannot be absolutely certain that some children do not have pneumonia solely from a non-bacterial cause.

- Choosing amoxicillin-clavulanate may limit the generalisability of results in settings where amoxicillin is generally used to treat pneumonia.

to-next respiratory-related hospitalisation and antibiotic resistance of nasopharyngeal respiratory bacteria. Ethics and dissemination The Human Research Ethics Committees of all the recruiting institutions (Darwin: Northern Territory Department of Health and Menzies School of Health Research; Auckland: Starship Children's and KidsFirst Hospitals; East Malaysia: Likas Hospital and Sarawak General Hospital; Kuala Lumpur: University of Malaya Research Ethics Committee; and Klang: Malaysian Department of Health) have approved the research protocol version 7 (13 August 2018). The RCT and other results will be submitted for publication.

Trial registration ACTRN12616000046404. 


\section{INTRODUCTION}

Acute lower respiratory infections (ALRIs), including pneumonia, are the greatest cause of global childhood morbidity and mortality beyond the neonatal period. ${ }^{12}$ Moreover, they are associated with future deficits in pulmonary function ${ }^{3}$ and chronic lung disease, including bronchiectasis ${ }^{4}$ and chronic obstructive pulmonary disease (COPD). ${ }^{5}$ Bronchiectasis and non-smoking related $\mathrm{COPD}^{5}$ are both highly prevalent in disadvantaged 'at risk populations' encountered in low-to-middle income countries ${ }^{6}$ and among Indigenous peoples. ${ }^{78}$ Our earlier case-control study among Indigenous children in Central Australia found that a history of hospitalised pneumonia significantly increased the risk of having bronchiectasis (OR 15.2, 95\% CI 4.4 to 52.7). ${ }^{4}$ Furthermore, both COPD and bronchiectasis are associated with early mortality where, for example, bronchiectasis causes premature death in the third to fourth decade of life in Australian Indigenous adults. ${ }^{9}$ Yet, many knowledge gaps in managing pneumonia remain, especially the optimal duration of antibiotic treatment, ${ }^{10}$ while the full extent of the medium and long-term consequences of pneumonia are largely unknown. 21112

As the WHO diagnostic algorithm for pneumonia used in resource poor settings is designed to reduce mortality, it sacrifices specificity for sensitivity. Antibiotics are recommended for all those suspected of clinical pneumonia. In non-HIV infected children aged $<5$ years, 3 days of oral amoxicillin is recommended for all those with tachypnoea alone and is extended to 5 days if subcostal recession is also present. Those with severe clinical pneumonia (eg, dehydration, altered conscious state) receive 5 days of intravenous antibiotics (penicillin/ampicillin and gentamicin). ${ }^{1}$ These recommendations are based on several large randomised controlled trials (RCTs) of oral versus intravenous antibiotics and 3 versus 5 days of oral antibiotics conducted in children living in low-income countries. $^{1314}$ The adjusted failure rate on day- 6 in the four largest studies was $8.5 \%(95 \% \mathrm{CI} 5.9 \%$ to $11.5 \%){ }^{13}$ However, the external validity of these studies is limited and biased towards equivalence where neither treatment will be effective as most subjects diagnosed with clinical pneumonia likely had bronchiolitis or viral ALRIs because: (1) over half of the subjects were infants, (2) $20 \%$ to $80 \%$ had wheeze, (3) chest X-rays (CXR) were either not performed or abnormal in $<20 \%$ of cases and (4) oral amoxicillin and placebo produced equivalent outcomes in infants aged 2 to 59 months. ${ }^{15}$ In this context, the shorter courses of antibiotics recommended by WHO cannot be extrapolated to pneumonia that is likely to be caused by bacterial pathogens ${ }^{16}$ in children at high-risk of ongoing morbidity, the target group of our study.

In comparison, in CXR-confirmed hospitalised pneumonia, there is little information guiding treatment duration. A single-centre three-arm RCT involving 140 non-hospitalised children aged $<5$ years from Israel with likely bacterial pneumonia (based on clinical criteria, CXR, white blood cell counts) found that the failure rate of a 3 day course of amoxicillin $(80 \mathrm{mg} / \mathrm{kg} /$ day in three divided doses) in this patient group (40\%) was unacceptably high. ${ }^{17}$ In contrast, 5 and 10 days of oral amoxicillin using the same dosing regimen had equivalent outcomes (no failures). ${ }^{17}$ Consequently, the duration of antibiotic treatment recommended in national guidelines for pneumonia varies between low-income countries (WHO 3 to 5 days) and high-income countries (5 to 10 days). ${ }^{17} 18$ The few RCTs of antibiotic duration for treating child pneumonia report only short-term outcomes (eg, mortality, persistent fever and respiratory signs after 5 days, or relapse within 1 to 2 weeks with only one study following subjects for 30 days). ${ }^{141718}$ No studies have examined the duration of chronic symptoms (eg, wet cough >4 weeks) post-treatment, subsequent respiratory-associated hospitalisations or persistent CXR abnormalities following pneumonia. These outcomes beyond the 4 week time point are important as we ${ }^{19}$ and others ${ }^{20}$ have shown that chronic wet cough in children implies lower airway infection and intense inflammation, as seen in protracted bacterial bronchitis.

Longer antibiotic courses may be beneficial in selected settings. Although controversial, in adults with ventilator-associated pneumonia, a meta-analysis found evidence for increased risk of relapse with shorter antibiotic treatments (OR 1.7; 95\% CI 1.0 to 2.8) during the following 4 weeks and all clinical point estimates favoured longer treatment courses. ${ }^{21}$ Supportive meta-analysis and large RCT data in other bacterial infections (streptococcal tonsillitis, ${ }^{22}$ otitis media in children aged $<2$ years ${ }^{23} 24$ and urinary tract infections ${ }^{25}$ ) show higher recurrence, failure and/or poorer bacterial eradication rates with shorter ( $<7$ days) antibiotic courses. Thus, preventing persistent wet cough (indicating presence of infected secretions) and/or recurrent ALRIs or pneumonia after an initial episode of pneumonia could have long-term benefits for lung health in children from groups deemed to be at high-risk of these complications. ${ }^{26}$

Young 'at-risk' children, such as disadvantaged Indigenous children ${ }^{4}$ and those in low-income countries, are heavily colonised with respiratory bacterial pathogens from an early age and may benefit from a longer antibiotics course for severe pneumonia to increase the likelihood of pathogen clearance from their airways and to reduce the risk of persistent inflammation and airway injury. These young children have developing lungs (hence more susceptible to injury) ${ }^{27}$ and the presence of protracted low grade infection and inflammation postALRI could trigger the vicious cycle associated with bronchiectasis. $^{27} 28$ Thus, a longer antibiotic course for young children hospitalised with severe pneumonia could enhance bacterial clearance and reduce the risk of longterm complications.

We are therefore undertaking a RCT comparing 'longer' with standard ('shorter') antibiotic treatment courses for severe pneumonia. We are aware that longer antibiotic treatment for all children might increase antibiotic resistance and lead to 'over-treatment' of some children. Thus, 
we are also monitoring antibiotic resistance in respiratory bacterial pathogens from the nasopharynx.

\section{Study objectives and hypotheses}

Our primary question is: Does a longer (13 to 14 days) course of antibiotics, compared to a shorter (5 to 6 days) one, improve the long-term clinical outcome of children hospitalised with severe community-acquired, CXR-confirmed pneumonia? Our primary hypothesis is that a longer antibiotic course, compared with the standard treatment course reduces the risk of chronic respiratory symptoms/signs or bronchiectasis at 24 months after the original episode of pneumonia.

Our secondary aims are to:

1. Determine the effects of antibiotic course duration on:

(i) clinical cure rates (at 4 weeks), (ii) time-to-next hospitalisation for a respiratory illness and (iii) antibiotic resistance in respiratory pathogens isolated from the nasopharynx.

2. Identify the predictors of chronic respiratory symptoms or bronchiectasis, specifically the contribution of persistent CXR abnormalities 4 weeks following treatment for pneumonia.

Our secondary hypotheses are that: (1) a longer antibiotic course with amoxicillin-clavulanate compared with the standard treatment course (a) improves clinical cure rates at 4 weeks, (b) lengthens the time-to-next exacerbation and (c) does not increase antibiotic resistance in nasopharyngeal respiratory bacterial pathogens; and (2) children who develop chronic respiratory symptoms can be identified earlier by the persistence of CXR abnormalities 4 weeks after an episode of pneumonia.

\section{METHODS AND ANALYSIS}

\section{Study setting and design}

We are conducting a multicentre, parallel group, doubleblind placebo RCT (with concealed allocation) involving the paediatric departments of seven participating hospitals in six cities from three different countries: Royal Darwin Hospital in Darwin, Northern Territory (NT, Australia); Starship Children's Hospital and KidzFirst Hospital in Auckland (New Zealand) and four Malaysian cities (Likas Hospital in Kota Kinabalu, Sabah; Sarawak General Hospital in Kuching, Sarawak; University of Malaya Medical Centre in Kuala Lumpur, Wilayah Persekutuan and Hospital Tengku Ampuan Rahimah in Klang, Selangor). Our study design, summarised in figure 1, is in accordance with Standard Protocol Items: Recommendations for Interventional Trials guidelines. ${ }^{29}$ Our study is ongoing; we recruited our first participant on 30 May, 2016, (in Darwin) and expect to continue recruiting until late 2019 with the study anticipated to be completed by late 2021.

\section{Study population}

The inclusion criteria are:

1. Hospitalised children aged 3 months to $<6$ years.
2. Aboriginal and Torres Strait Islander children in Darwin, Māori or Pacifica (Pacific Islander) children in Auckland, and any child without ethnic restriction in Malaysia.

3. Features of severe pneumonia on admission (fever $\geq 37.5^{\circ} \mathrm{C}$, age-adjusted tachypnoea (respiratory rate $[R R] \geq 50$ if aged $\leq 12$ months; $R R \geq 40$ if aged $>12$ months) with chest wall recession and/or peripheral oxygen saturation $\left[\mathrm{SpO}_{2}\right]<92 \%$ in air ${ }^{30}$ and consolidation on CXR as evaluated by the treating clinician. $^{31}$

4. Becoming afebrile within 1 to 3 days of commencing intravenous antibiotics, with improved respiratory symptoms and signs, $\mathrm{SpO}_{2}>92 \%$ in air and are ready to be switched to oral amoxicillin-clavulanate.

5. Had symptoms for $\leq 7$ days at the time of admission to hospital.

Exclusion criteria are: (1) current wheeze (during hospitalisation), (2) underlying co-morbidities other than asthma (eg, bronchiectasis, cyanotic congenital heart disease, cardiac failure, neuromuscular disorders, immunodeficiency) that could potentially influence the current illness, (3) severe malnutrition (weight-for-height Z-score <-3.0), (4) complicated pneumonia (effusion, empyema or abscess, tuberculosis) and/or where pathogens are identified that require different antibiotics or extended courses of treatment (eg, Staphylococcus aureus, Burkholderia pseudomallei, Mycobacterium tuberculosis or Mycoplasma pneumoniae), (5) concurrent extra-pulmonary infection requiring additional antibiotic therapy (eg, meningitis), (6) penicillin allergy, (7) enrolled in the study previously or (8) unable to return for follow-up clinic visits during the next 24 months.

\section{Recruitment}

Eligible children with radiographic-confirmed pneumonia are identified by a member of the research team visiting the paediatric wards of each hospital twice daily. Within 24 hours of admission to the paediatric ward, the parent/ guardian of a potentially eligible child is approached. A member of the research team discusses the study with the parent/guardian in detail using the consent flip chart, information booklet and interpreters (where appropriate). After all aspects of study are explained, including potential risks and benefits, written informed consent is obtained from parents/guardians who agree to participate in the study. Each site maintains a screening log with limited details recorded (screening number, date, age and sex of the person, whether informed consent was obtained or reason for refusal [if the participant wishes to provide that information]).

During hospitalisation, children with uncomplicated segmental or lobar pneumonia receive 1 to 3 days of intravenous site-specific antibiotics (either ampicillin or benzylpenicillin $\mathrm{G}$ according to the hospital's protocol) and oxygen supplementation if $\mathrm{SpO}_{2}$ in air $\leq 92 \%$. Other clinical management (eg, intravenous fluids, antipyretics, nutritional support) is at the discretion of the treating 


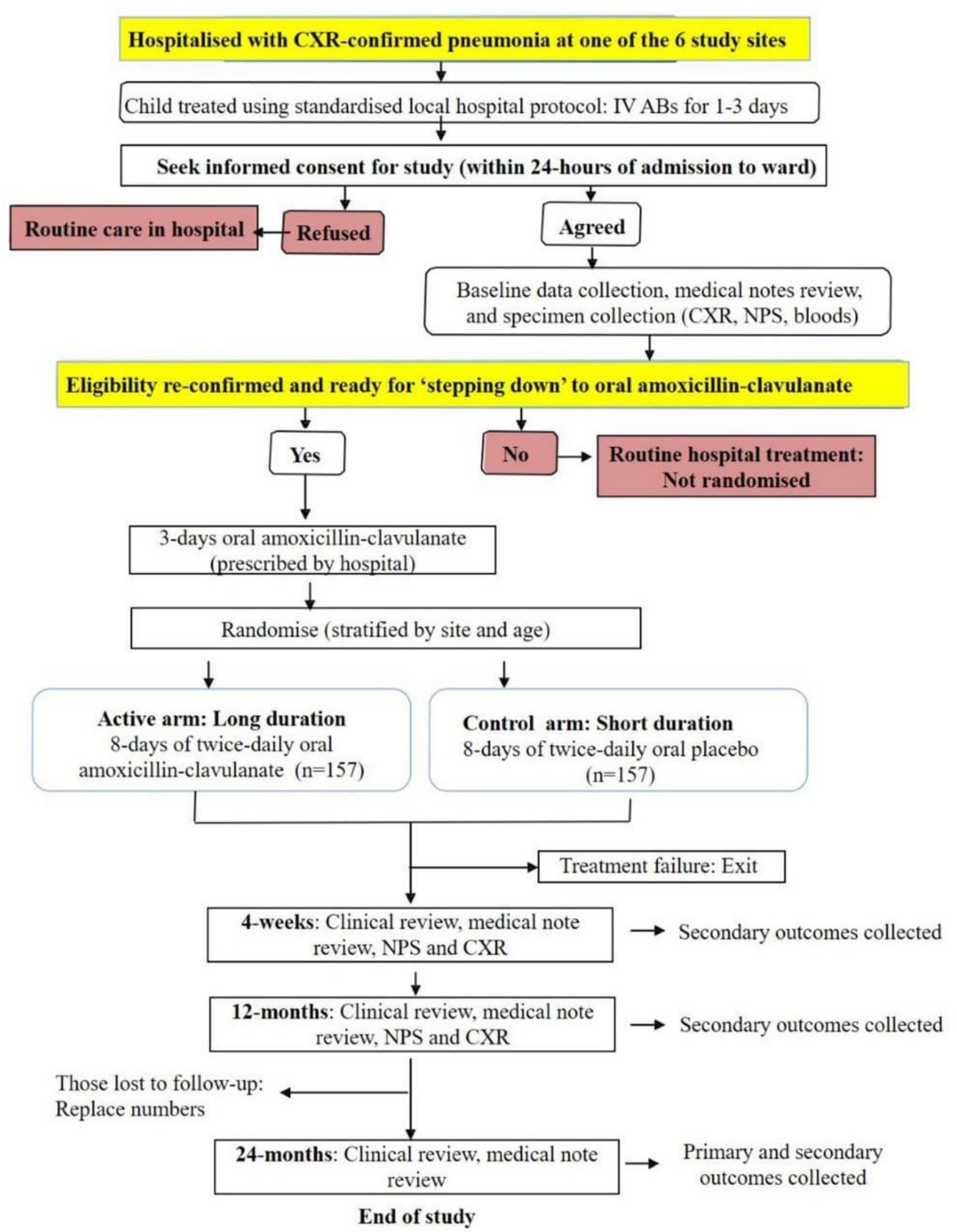

Figure 1 Overall schematic study design. ABs, antibiotics; CXR, chest X-ray; NPS, nasopharyngeal swab.

paediatrician. The eligibility of children who have been consented is reassessed when they are ready to 'step down' from intravenous to oral antibiotics. In children not eligible for randomisation (ie, not meeting the full eligibility criteria), the parent/guardian is advised that their child will be managed in accordance with the treating hospital team's routine pneumonia protocol. Those meeting the inclusion criteria are then enrolled into the RCT and randomised to one of the two treatment arms that follow on from the 3 days of oral amoxicillin-clavulanate received by all trial participants.

\section{Randomisation and allocation}

The randomisation sequence was computer generated and used random permuted block sizes of 4 to 8 , prepared by a statistician external to the study team. Treatment groups are allocated according to the randomisation code. On enrolment, the child is assigned to the next unique number on the appropriate stratified list. The allocation is performed by the trial pharmacist at the respective hospitals. Randomisation is stratified by city site (Darwin, Auckland, Kota Kinabalu, Kuching, Kuala Lumpur, Klang) and age ( $<1$ or 1 to 5 years). The 
allocation sequence is concealed and will not be revealed until data analyses are complete.

\section{Treatment groups and blinding}

After ceasing intravenous antibiotics and completing the 3 days of oral amoxicillin-clavulanate 400/57 duo suspension formulation $(80 \mathrm{mg} / \mathrm{kg} /$ day of the amoxicillin component in two divided doses), the children randomised to one of two treatment groups will receive either: (1) in the active arm: 8 days of oral amoxicillin-clavulanate $400 / 57$ duo suspension $(80 \mathrm{mg} / \mathrm{kg}$ / day of amoxicillin component in two divided doses; $\max 980 \mathrm{mg}$ per day) or (2) in the control arm: 8 days of oral placebo suspension (in two divided doses in volumes equal to the active arm). The placebo medication, manufactured specifically by the Institute of Drug Technology Australia Limited (Melbourne, Victoria), has an indistinguishable taste, smell, texture and appearance to the active medication, as used previously. ${ }^{32}$ Both the active antibiotic and placebo are in matched opaque bottles. For all trial medications, equal volumes of water are added using a syringe in a child-safety, sealed, opaque bottle. Thus, blinding of the child's allocation group will be maintained throughout the trial until the analyses is complete. Unblinding will be only permissible if a serious adverse event occurs and we are requested by the independent data monitoring committee (iDMC) to unblind the child's allocated group.

\section{Data collection}

Data are collected from the parent/guardian and from the medical records at baseline (enrolment), 4weeks, 12 months and 24 months post-hospital discharge. On enrolment, demographic information (age, sex, ethnicity, household size, etc), birth history, breast-feeding history, prior illness, immunisation record, in-utero and household smoke exposure are recorded, and a physical examination performed by trained research staff. Immunisation data are collected from the respective country's immunisation register or patients' records. All data are documented on hard copy standardised forms and entered into the database at a later date. De-identified data in the central database is password protected and confidentiality maintained. Electronic forms are not used because of the instability of the internet connection at some sites. The final dataset will be accessible to each site's principal investigators, the grant holders, iDMC and the study statistician.

As with our previous RCTs, ${ }^{33} 34$ children are prospectively followed after being discharged from hospital. In the current study, follow-up is for 24 months post-hospital discharge with clinical reviews at the hospital or local health clinics at 4 weeks, 12 months and 24 months. The primary and secondary outcome measures are collected at the time points below. Serious and non-serious (nausea, vomiting, diarrhoea, rash) adverse-events are documented and monitored. Safety exit points are discussed below. Adherence is assessed by parent report and return of empty bottles.

\section{Specimen collection}

At enrolment (baseline), as close as possible to the day of admission, and again at 4 weeks and 12 months following hospital admission for pneumonia, all children will have nasopharyngeal swabs taken, which are placed immediately into skim milk tryptone glucose glycerol broth medium and kept cold until stored at $-80^{\circ} \mathrm{C}$.

Routine blood collection (ie, full blood count and blood cultures) are undertaken by the treating hospital medical team on admission and these data are recorded. Also, CXRs are performed at admission (in all cases) and at 4 weeks and 12 months when possible (ie, some children living in remote regions are unable to travel to the treating hospital for a CXR).

\section{Treatment failure and exit criteria during the study}

Monitoring of adverse and serious adverse events are undertaken throughout the trial using standardised adverse and serious adverse event forms. Treatment failure and exit criteria are defined as occurrence within 4 weeks of enrolment of any of the following: (1) death, (2) secondary fever $\left(>38^{\circ} \mathrm{C}\right)$ with new signs of clinical pneumonia or (3) new CXR changes. Participants are discontinued from the study protocol if the parent/ guardian withdraws consent for participation, the child or parent/guardian are unable to comply with study procedures or if a trial medication related serious adverse event or exit criterion occurs.

\section{Outcome measures}

Our primary outcome is the proportion of children without chronic respiratory symptoms/signs or bronchiectasis $^{35}$ at 24 months following hospitalisation for pneumonia. Our secondary outcomes are: (1) the proportion with clinical cure (ie, complete resolution of respiratory symptoms and signs) at 4 weeks, (2) time-to-next respiratory-related hospitalisation, (3) CXR abnormalities and (4) adverse events. Our secondary laboratory outcomes are nasopharyngeal respiratory bacterial pathogens and antibiotic resistance (4 weeks, 12 months).

Similar to our other intervention RCTs, ${ }^{32-34} 36$ medical record review at community health centres and each of the hospital sites will identify respiratory re-hospitalisation episodes and prior investigations for chronic lung diseases, including radiographic investigations for bronchiectasis. Additionally, parents will be specifically asked about re-hospitalisations and whether a chest CT scan has been undertaken, which will be verified with checking medical records.

CXRs are digitised and anonymised. These will be scored independently by two people (respiratory physicians or radiologist) in a blinded manner. CXR abnormalities on follow-up will be dichotomised into abnormal (presence of new or persistent parenchymal densities or 
infiltrates) $)^{31} 37$ or normal. Disagreements will be resolved by consensus.

The stored nasopharyngeal swabs will be processed as done previously. ${ }^{3236}$ Briefly, batches of swabs will be thawed and cultured on selective media. Streptococcus pneumoniae, Haemophilus influenzae, Moraxella catarrhalis and S. aureus will be confirmed by standard techniques. ${ }^{36}{ }^{38}$ Based on their colonial morphology, four isolates each of S. pneumoniae, $H$. influenzae ${ }^{38}$ and $S$. aureus will have their antibiotic susceptibilities determined by the calibrated susceptibility disc diffusion method. ${ }^{36}$ The minimum inhibitory concentration for penicillin for $S$. pneumoniae will be determined by E-tests if the oxacillin disc zone is $<6 \mathrm{~mm}$ and for $H$. influenzae if the ampicillin disc zone is $<6 \mathrm{~mm} .{ }^{36}$ The European Committee on Antimicrobial Susceptibility Testing criteria will identify resistant isolates. A nitrocephin-based test will detect beta-lactamase activity in $H$. influenzae and M. catarrhalis isolates.

\section{Sample size}

Sample size estimates are based on our primary outcome (proportion without chronic respiratory symptoms/ signs or bronchiectasis at 24 months). We wish to detect a significant difference between the active arm (ie, longer antibiotic course) and the control arm (ie, shorter antibiotic course). We assume the percentage without chronic respiratory morbidity (at 24 months) in the short antibiotic group (controls) is $65.9 \%$ (based on our previous Central Australian pneumonia study ${ }^{31}$ where $34.1 \%$ had persistent respiratory morbidity at 12 months). To find a $50 \%$ reduction in bronchiectasis (or chronic respiratory symptoms), the percentage without bronchiectasis in the long antibiotic group will be $82.9 \%$. For $89 \%$ power and alpha $=0.05$, we require outcome data from 131 patients in each treatment group. Adding 20\% (for participant drop-out), we will enrol 157 children in each group. To mitigate the possibility of an even higher drop-out rate, we will replace participants that missed the 12 month follow-up, until late 2019. Replacements will be assigned a new identification number and treatment allocation.

\section{Statistical analyses and reporting}

Data coding and entry is based at Darwin and conducted in accordance with good clinical practice. Data will be reported and presented in accordance with the updated Consolidated Standards of Reporting Trials criteria. ${ }^{39}$ Children will be analysed according to the intention-totreat principle where analysis will be by allocation status regardless of subsequent management. A detailed statistical analysis plan that is approved by the iDMC will be in place prior to data analyses.

For our primary aim, the main effects of the intervention will be determined by comparing the proportion without any chronic respiratory symptoms/signs or evidence of bronchiectasis at 24 months. We will report relative risk calculated via log-binomial regression models. Generalised linear models will be used to calculate absolute differences between proportions (with 95\% CI), as in previous analyses. ${ }^{32} 36$

For our secondary aims, the effects of the interventions will be determined by: (1) comparing the proportion with clinical cure (complete resolution of respiratory symptoms and signs) at 4 weeks and (2) constructing a Kaplan-Meier curve for each group for time-to-next respiratory-related hospitalisation, and proportions will be compared using the log-rank test as well as time-to-event regression models (as above). Logistic regression will be used to examine the effects of treatment duration on respiratory bacterial pathogen colonisation in the nasopharynx and antibiotic resistance with models adjusted for potentially confounding demographic, social and clinical variables as appropriate, as done previously. ${ }^{32} 36$ CXR abnormality data will be compared between children with and without chronic respiratory symptoms/ bronchiectasis. CXR abnormalities will be dichotomised (present/absent) and $\chi^{2}$ tests used to determine the OR with $95 \%$ CIs for presence of chronic symptoms/ bronchiectasis.

\section{Patient and public involvement}

The study was discussed with, and approved by, the Indigenous Reference Group (Darwin) prior to submitting the grant applications and beginning the study. Also, the study was discussed with, and approved by, the Māori Research Review Committee (Auckland) prior to submitting the New Zealand based grant application and commencement of the study in New Zealand in 2017. Patients were not involved in the study question or design, but the above committees members have family members who have had pneumonia as children, and they approved of the study design and outcome measures.

\section{Ethics, dissemination and safety monitoring}

We will publish the results in a major medical journal (using the International Committee of Medical Journal Editors [ICMJE] author guidelines), and share the outcomes with the academic and medical community, funding and relevant patient organisations. Professional writers will not be used. All adverse events, including serious adverse events, are monitored by an iDMC, which was established and met prior to commencing the study. An Indigenous Reference Group oversees the cultural aspects of the study.

\section{DISCUSSION}

We are currently undertaking a multicentre, double-blind RCT in three countries to address the question of whether a longer course of antibiotics (13 to 14 days), compared with the standard short course ( 5 to 6 days), for treating young children hospitalised with community-acquired pneumonia (assumed by fulfilling the inclusion criteria to be of bacterial origin) reduces its long-term complications. The biological plausibility of our hypothesis was 
presented above. The outcomes and time points were chosen carefully as described below.

Many factors influence the prevalence of infectious agents associated with pneumonia. These include: (a) patient factors (eg, age, nutritional state, co-morbidities, immunisations received), (b) the timing, site and nature of specimens taken, (c) the type and depth of laboratory tests used and (d) the nature and intensity of exposure to circulating microorganisms (eg, crowding, household and external air pollution, seasons, community use of antibiotics). ${ }^{11}{ }^{40}$ As our study settings differ, it is unknown whether bacterial causes of pneumonia vary between the sites. Nevertheless, in the context of the difficulty in ascribing aetiology of pneumonia when blood cultures are negative and without direct lung samples, we did not aim to undertake an aetiology study.

\section{Rationale for our chosen outcome measures and time frame}

Chronic cough (defined as $>4$ weeks duration ${ }^{41}{ }^{42}$ ) can occur post-ALRIs. ${ }^{43}$ In children, chronic cough has an often unrecognised high morbidity, ${ }^{44}$ may reflect the underlying cause, ${ }^{45}$ and is the dominant symptom of bronchiectasis. ${ }^{27}$ In a national study, we found that $9 \%$ of 346 children newly referred for chronic cough had bronchiectasis, which was significantly more likely in Indigenous than non-Indigenous children (OR 4.4, $95 \%$ CI 1.9 to 10.0$).^{45}$ Further, our follow-up study of young children hospitalised for bronchiolitis identified that the persistence of cough at 3 to 4 weeks post-discharge significantly increased the OR of having bronchiectasis detected at a median of 13 months post-index hospitalisation. ${ }^{46}$ Also, our earlier study observed that of 109 Indigenous Northern Territory children hospitalised with CXR-proven pneumonia, of whom 90 (83\%) were reviewed at 12 months, $23 / 90(26 \%)$ had a new treatable chronic respiratory illness. ${ }^{31}$ Finally, in a New Zealand study, ${ }^{47} 94 / 164$ (74\%) (86\% Māori/Pacifica) infants and young children hospitalised before age 2 years for bronchiolitis $(44 \%)$ or pneumonia $(56 \%)$ underwent a clinical review 12 months later where 81 also had a repeat CXR. Overall, in the follow-up cohort 29/94 (31\%) had a chronic wet cough history, 30 (32\%) still had a cough with or without chest crackles on examination and $50 / 81(62 \%)$ had pulmonary infiltrates present. ${ }^{47}$ Thus, we chose these chronic cough, evaluated at 4 weeks, 12 months and 24 months post-hospitalisation for pneumonia, and CXR changes at the two earlier timepoint, as they are important markers for future lung health.

\section{Rationale for amoxicillin-clavulanate}

Amoxicillin is the recommended oral antibiotic for treating non-severe pneumonia in young children aged $<5$ years from both high income and low-to-middle income countries. ${ }^{130}$ Our original intention was to use amoxicillin as 'step-down' therapy in the RCT, but unfortunately the cost of preparing and manufacturing its placebo proved prohibitive. Instead, we chose amoxicillin-clavulanate, since from our previous studies it was well tolerated and importantly we had both access and guaranteed supply of a validated placebo medication, indistinguishable from the active antibiotic. ${ }^{32}{ }^{48}$ Because of its broader-spectrum of action, amoxicillin-clavulanate is recommended as a second-line agent for inpatient and outpatient treatment of pneumonia. ${ }^{130}$ Nevertheless, a recent survey of 19 hospitals in 16 European countries identified it was used as a first-line antibiotic by more than $25 \%$ of centres for treating paediatric community-acquired pneumonia in both hospital and community settings. ${ }^{49}$ Amoxicillin-clavulanate offers the theoretical advantage of activity against $\beta$-lactamase producing strains of $H$. influenzae and M. catarrhalis that heavily colonise the upper airways of these young children and may play a role in establishing chronic lung disease following an episode of severe pneumonia. ${ }^{412}$ The accompanying risk of course is selecting antibiotic-resistant organisms. To this end we are monitoring antibiotic susceptibility patterns of nasopharyngeal respiratory bacterial pathogens 4 weeks and 12 months after commencing treatment for pneumonia. The nasopharynx was chosen because of its relative ease of sampling access and as it acts as reservoir for transmitting antibiotic-resistant respiratory bacterial pathogens within the community. ${ }^{50}$

In summary, our study addresses a large knowledge gap of a common worldwide problem in early childhood pneumonia. The multicentre nature of our study increases the generalisability of the future findings of our RCT that is complemented by assessing predictors of future morbidity.

\section{Author affiliations}

${ }^{1}$ Child Health Division, Menzies School of Health Research, Charles Darwin Univ, Darwin, Northern Territory, Australia

${ }^{2}$ Qld Children's Hospital, Brisbane, Queensland, Australia

${ }^{3}$ Hospital Likas, Kota Kinabalu, Malaysia

${ }^{4}$ Global and Tropical Health Division, Menzies School of Health Research, Darwin, Northern Territory, Australia

${ }^{5}$ School of Population Health, The University of Queensland, Brisbane, Queensland, Australia

${ }^{6}$ Menzies School of Health Research, Charles Darwin University, Darwin, Northern Territory, Australia

${ }^{7}$ University of Malaya, Kuala Lumpur, Malaysia

${ }^{8}$ Universiti Malaysia Sarawak, Kuching, Malaysia

${ }^{9}$ Paediatrics, Univeristy of Malaya, Kuala Lumpur, Malaysia

${ }^{10}$ Starship Children's Hospital, Auckland, New Zealand

${ }^{11}$ Charles Darwin University, Darwin, Northern Territory, Australia

${ }^{12}$ Tengku Ampuan Rahimah Hospital, Klang, Malaysia

${ }^{13}$ Prince Alfred Hospital, Sydney, New South Wales, Australia

${ }^{14}$ Child Health Division, Menzies School of Health Research, Darwin, Northern Territory, Australia

${ }^{15}$ Menzies School of Health Research, Darwin, Northern Territory, Australia

${ }^{16}$ School of Medicine, The University of Queensland, Brisbane, Queensland, Australia

${ }^{17}$ Menzies Health Institute Queensland, Griffith University, Southport, Queensland, Australia

Acknowledgements We thank the research staff (Blueren Wilde, Lesley Versteegh, Erin Plumb, Charmaine Mobberley, Cate Wilson, Francisca Tenorio, Shirley Lawrence) for facilitating the study and Anita Champion for assistance in obtaining the medications. We are also grateful to members of the Indigenous Reference Group of the Child Health Division at Menzies School of Health Research (Bilawara Lee, Mark Mayo, Tracey Brand, Christine Campbell, Dennis Bonney, Lorna Murakami-Gold, Pirrawayinyi Puruntatameri, Veronica McClintic, Lesley Versteegh, 
Loyla Leysley) for supporting this study and for over-seeing the cultural aspects. We also thank Prof Alan Isles, Dr Kok Meng John Chan, Professor Lucy Lum and Dr Carolyn Maclennan for voluntarily providing their time as members of the independent data monitoring committee.

Contributors $\mathrm{ABC}$ conceived and designed the study, drafted the manuscript and was primarily responsible for obtaining the grant. KG co-conceived and designed the study, had a major input into the grant submission and edited the manuscript. RSW led the statistical components for grant submission. JWU, SMF, TWY, MHO AMN, JdB, PSM, BL, PT contributed to study design, grant submission and manuscript. $C A B$ is the lead investigator at the Auckland site and obtained a 2-year grant for recruiting the children in New Zealand. SMF is the lead investigator in Kota Kinabalu, MHO is the lead investigator in Kuching, AMN is the lead investigator in Kuala Lumpur and obtained a Malaysian grant that supported the study in Kuala Lumpur. NN and NS are the lead investigators at the Klang site. GBM initiated the project, prepared the SOPs, submitted the ethics application and is currently coordinating all aspects of the project. HSV leads the laboratory component of assessing nasopharyngeal bacteria and its resistance to antibiotics. All authors read and approved the final manuscript.

Funding The study is funded by a 5 -year Australian National Health and Medical Research Council (NHMRC) project grant (number 1098443) and supported by a NHMRC Centre for Research Excellence in Lung Health of Aboriginal and Torres Strait Islander Children (grant number 1040830). The New Zealand site is supported by a 2-year grant from CureKids, New Zealand (grant 3571). The Kuala Lumpur site is partially funded by a University Malaya Research Grant RP026-14HTM. AC is supported by a NHMRC practitioner fellowship (grant 1058213). GM is supported by a NHMRC early career fellowship (grant 1111705). The views expressed in this publication are those of the authors and do not reflect the views of the NHMRC. The primary grant is held by the Menzies School of Health Research (Darwin), which also acts as the trial sponsor.

Competing interests None declared.

Patient consent for publication Not required.

Provenance and peer review Not commissioned; externally peer reviewed.

Data sharing statement Data sharing among institutions is in accordance to the Australian NHMRC multi-institutional agreement.

Open access This is an open access article distributed in accordance with the Creative Commons Attribution Non Commercial (CC BY-NC 4.0) license, which permits others to distribute, remix, adapt, build upon this work non-commercially, and license their derivative works on different terms, provided the original work is properly cited, appropriate credit is given, any changes made indicated, and the use is non-commercial. See: http://creativecommons.org/licenses/by-nc/4.0/.

\section{REFERENCES}

1. WHO. Revised WHO classification and treatment of childhood pneumonia: WHO, 2014

2. Madhi SA, De Wals P, Grijalva CG, et al. The burden of childhood pneumonia in the developed world: a review of the literature. Pediatr Infect Dis J 2013;32:e119-27.

3. Chan JY, Stern DA, Guerra S, et al. Pneumonia in childhood and impaired lung function in adults: a longitudinal study. Pediatrics 2015;135:607-16.

4. Valery PC, Torzillo PJ, Mulholland K, et al. Hospital-based casecontrol study of bronchiectasis in indigenous children in Central Australia. Pediatr Infect Dis J 2004;23:902-8.

5. Salvi SS, Barnes PJ. Chronic obstructive pulmonary disease in nonsmokers. Lancet 2009;374:733-43.

6. McCallum GB, Binks MJ. The epidemiology of chronic suppurative lung disease and bronchiectasis in children and adolescents. Front Pediatr 2017:5:27.

7. O'Grady KA, Revell A, Maguire GP, et al. Lung health care for Aboriginal and Torres Strait Islander Queenslanders: breathing easy is not so easy. Aust Health Rev 2011;35:512-9.

8. Basnayake TL, Morgan LC, Chang AB. The global burden of respiratory infections in indigenous children and adults: a review. Respirology 2017;22:1518-28.

9. Blackall SR, Hong JB, King P, et al. Bronchiectasis in indigenous and non-indigenous residents of Australia and New Zealand. Respirology 2018;23:743-9.

10. Grimwood K, Fong SM, Ooi MH, et al. Antibiotics in childhood pneumonia: how long is long enough? Pneumonia 2016;8:6.
11. Chang $A B$, Ooi MH, Perera $D$, et al. Improving the diagnosis, management, and outcomes of children with pneumonia: where are the gaps? Front Pediatr 2013;1:29.

12. Grimwood K, Chang AB. Long-term effects of pneumonia in young children. Pneumonia 2015;6:101-14.

13. Fox MP, Thea DM, Sadruddin S, et al. Low rates of treatment failure in children aged 2-59 months treated for severe pneumonia: a multisite pooled analysis. Clin Infect Dis 2013;56:978-87.

14. Lassi ZS, Das JK, Haider SW, et al. Systematic review on antibiotic therapy for pneumonia in children between 2 and 59 months of age. Arch Dis Child 2014;99:687-93.

15. Hazir T, Nisar YB, Abbasi S, et al. Comparison of oral amoxicillin with placebo for the treatment of world health organization-defined nonsevere pneumonia in children aged 2-59 months: a multicenter, double-blind, randomized, placebo-controlled trial in pakistan. Clin Infect Dis 2011;52:293-300.

16. Ben-Shimol S, Levy-Litan V, Falup-Pecurariu O, et al. Evidence for short duration of antibiotic treatment for non-severe community acquired pneumonia (CAP) in children - are we there yet? A systematic review of randomised controlled trials. Pneumonia 2014;4:16-23.

17. Greenberg D, Givon-Lavi N, Sadaka Y, et al. Short-course antibiotic treatment for community-acquired alveolar pneumonia in ambulatory children: a double-blind, randomized, placebo-controlled trial. Pediatr Infect Dis J 2014;33:136-42.

18. Kerrison C, Riordan FA. How long should we treat this infection for? Arch Dis Child Educ Pract Ed 2013;98:136-40.

19. Chang AB, Redding GJ, Everard ML. Chronic wet cough: protracted bronchitis, chronic suppurative lung disease and bronchiectasis. Pediatr Pulmonol 2008;43:519-31.

20. Douros K, Alexopoulou E, Nicopoulou A, et al. Bronchoscopic and high-resolution CT scan findings in children with chronic wet cough. Chest 2011;140:317-23.

21. Dimopoulos G, Poulakou G, Pneumatikos IA, et al. Short- vs longduration antibiotic regimens for ventilator-associated pneumonia: a systematic review and meta-analysis. Chest 2013;144:1759-67.

22. Falagas ME, Vouloumanou EK, Matthaiou DK, et al. Effectiveness and safety of short-course vs long-course antibiotic therapy for group a beta hemolytic streptococcal tonsillopharyngitis: a metaanalysis of randomized trials. Mayo Clin Proc 2008;83:880-9.

23. Paradise JL. Short-course antimicrobial treatment for acute otitis media: not best for infants and young children. JAMA 1997;278:1640-2.

24. Hoberman A, Paradise JL, Rockette HE, et al. Shortened antimicrobial treatment for acute otitis media in young children. $N$ Engl J Med 2016;375:2446-56.

25. Keren R, Chan E. A meta-analysis of randomized, controlled trials comparing short- and long-course antibiotic therapy for urinary tract infections in children. Pediatrics 2002;109:e70.

26. Singleton RJ, Valery PC, Morris $P$, et al. Indigenous children from three countries with non-cystic fibrosis chronic suppurative lung disease/bronchiectasis. Pediatr Pulmonol 2014;49:189-200.

27. Chang $A B$, Bush A, Grimwood K. Bronchiectasis in children: diagnosis and treatment. Lancet 2018;392:866-79.

28. Chang AB, Marsh RL, Upham JW, et al. Toward making inroads in reducing the disparity of lung health in Australian indigenous and new zealand māori children. Front Pediatr 2015;3:9.

29. Chan AW, Tetzlaff JM, Gøtzsche PC, et al. SPIRIT 2013 explanation and elaboration: guidance for protocols of clinical trials. BMJ 2013;346:e7586.

30. Nascimento-Carvalho CM, Madhi SA, O'Brien KL. Review of guidelines for evidence-based management for childhood community-acquired pneumonia in under-5 years from developed and developing countries. Pediatr Infect Dis J 2013;32:1281-2.

31. Chang AB, Masel JP, Boyce NC, et al. Respiratory morbidity in central Australian Aboriginal children with alveolar lobar abnormalities. Med J Aust 2003;178:490-4.

32. Goyal V, Grimwood K, Byrnes CA, et al. Amoxicillin-clavulanate versus azithromycin for respiratory exacerbations in children with bronchiectasis (BEST-2): a multicentre, double-blind, non-inferiority, randomised controlled trial. Lancet 2018;392:1197-206.

33. McCallum GB, Morris PS, Grimwood K, et al. Three-weekly doses of azithromycin for indigenous infants hospitalized with bronchiolitis: a multicentre, randomized, placebo-controlled trial. Front Pediatr 2015;3:32.

34. McCallum GB, Morris PS, Chatfield MD, et al. A single dose of azithromycin does not improve clinical outcomes of children hospitalised with bronchiolitis: a randomised, placebo-controlled trial. PLoS One 2013;8:e74316.

35. Chang AB, Van Asperen PP, Glasgow N, et al. Children with chronic cough: when is watchful waiting appropriate? development of 
likelihood ratios for assessing children with chronic cough. Chest 2015;147:745-53.

36. Valery PC, Morris PS, Byrnes CA, et al. Long-term azithromycin for Indigenous children with non-cystic-fibrosis bronchiectasis or chronic suppurative lung disease (Bronchiectasis Intervention Study): a multicentre, double-blind, randomised controlled trial. Lancet Respir Med 2013;1:610-20.

37. Redding G, Singleton R, Lewis $\mathrm{T}$, et al. Early radiographic and clinical features associated with bronchiectasis in children. Pediatr Pulmonol 2004;37:297-304.

38. Chang AB, Grimwood K, White AV, et al. Randomized placebocontrolled trial on azithromycin to reduce the morbidity of bronchiolitis in Indigenous Australian infants: rationale and protocol. Trials 2011;12:94.

39. Schulz KF, Altman DG, Consort MD. Statement: updated guidelines for reporting parallel group randomised trials. Trials 2010;2010:32.

40. GBD 2016 Lower Respiratory Infections Collaborators. Estimates of the global, regional, and national morbidity, mortality, and aetiologies of lower respiratory infections in 195 countries, 1990-2016: a systematic analysis for the Global Burden of Disease Study 2016. Lancet Infect Dis 2018;18.

41. Chang AB, Oppenheimer JJ, Weinberger MM, et al. Use of management pathways or algorithms in children with chronic cough: CHEST Guideline and expert panel report. Chest 2017;151:875-83.

42. Chang AB, Landau LI, Van Asperen PP, et al. Cough in children: definitions and clinical evaluation. Med J Aust 2006;184:398-403.
43. O'Grady KF, Drescher BJ, Goyal V, et al. Chronic cough postacute respiratory illness in children: a cohort study. Arch Dis Child 2017;102:1044-8.

44. Chang AB, Upham JW, Masters IB, et al. Protracted bacterial bronchitis: the last decade and the road ahead. Pediatr Pulmonol 2016;51:225-42.

45. Chang AB, Robertson CF, Van Asperen PP, et al. A multicenter study on chronic cough in children: burden and etiologies based on a standardized management pathway. Chest 2012;142:943-50.

46. McCallum GB, Chatfield MD, Morris PS, et al. Risk factors for adverse outcomes of Indigenous infants hospitalized with bronchiolitis. Pediatr Pulmonol 2016;51:613-23.

47. Trenholme AA, Byrnes CA, McBride C, et al. Respiratory health outcomes 1 year after admission with severe lower respiratory tract infection. Pediatr Pulmonol 2013;48:772-9.

48. Marchant J, Masters IB, Champion A, et al. Randomised controlled trial of amoxycillin clavulanate in children with chronic wet cough. Thorax 2012;67:689-93.

49. Usonis V, Ivaskevicius R, Diez-Domingo J, et al. Comparison between diagnosis and treatment of community-acquired pneumonia in children in various medical centres across Europe with the United States, United Kingdom and the World Health Organization guidelines. Pneumonia 2016;8:5.

50. Serisier DJ. Risks of population antimicrobial resistance associated with chronic macrolide use for inflammatory airway diseases. Lancet Respir Med 2013;1:262-74. 